\title{
Article \\ BMP7 Functions to Regulate Proliferation of Dermal Papilla Cells in Hu Sheep
}

\author{
Yue $\mathrm{Li}^{1,+}{ }^{\text {, }}$ Xiaoyang Lv ${ }^{2,+}$, Shanhe Wang ${ }^{1}$, Xiukai Cao ${ }^{2}{ }^{\oplus}$, Zehu Yuan ${ }^{2}$, Tesfaye Getachew ${ }^{3}$, Joram M. Mwacharo ${ }^{3}$, \\ Aynalem Haile ${ }^{3}$ and Wei Sun ${ }^{1,2, *}$ \\ 1 College of Animal Science and Technology, Yangzhou University, Yangzhou 225009, China; \\ yzudkly2019@163.com (Y.L.); shanhe12315@163.com (S.W.) \\ 2 Joint International Research Laboratory of Agriculture and Agri-Product Safety of Ministry of Education \\ of China, Yangzhou University, Yangzhou 225009, China; dx120170085@yzu.edu.cn (X.L.); \\ cxkai0909@163.com (X.C.); yuanzehu1988@163.com (Z.Y.) \\ 3 International Centre for Agricultural Research in the Dry Areas, Addis Ababa 999047, Ethiopia; \\ T.Getachew@cgiar.org (T.G.); j.mwacharo@cgiar.org (J.M.M.); a.haile@cgiar.org (A.H.) \\ * Correspondence: sunwei@yzu.edu.cn \\ + These authors contributed equally to this work.
}

Citation: Li, Y.; Lv, X.; Wang, S.; Cao, X.; Yuan, Z.; Getachew, T.; Mwacharo, J.M.; Haile, A.; Sun, W. BMP7 Functions to Regulate Proliferation of Dermal Papilla Cells in Hu Sheep. Genes 2022, 13, 201. https: / / doi.org/ 10.3390 /genes 13020201

Academic Editors: Ran Di and Qiuyue Liu

Received: 27 December 2021

Accepted: 19 January 2022

Published: 22 January 2022

Publisher's Note: MDPI stays neutral with regard to jurisdictional claims in published maps and institutional affiliations.

Copyright: (C) 2022 by the authors. Licensee MDPI, Basel, Switzerland. This article is an open access article distributed under the terms and conditions of the Creative Commons Attribution (CC BY) license (https:// creativecommons.org/licenses/by/ $4.0 /)$.

\begin{abstract}
Bone morphogenetic proteins (BMPs) are the structurally similar and highly conserved type of functional proteins that play an important role in hair follicle growth and development. $B M P 7$ was a differentially expressed gene in different patterns of Hu sheep lambskin identified using Agilent microarray. Since hair follicle is the basis of pattern formation of lambskin, and its growth and development is governed by dermal papilla cells (DPCs), to clarify the role of BMP7 and hair follicle, our study was designed to investigate the regulation between BMP7 and DPCs. Firstly, the CDS region of BMP7 was cloned by 3'Race and PCR in Hu sheep and performed serious of bioinformatic analysis. Then, the effects of BMP7 on DPCs were analyzed after overexpression and interference of $B M P 7$ in dermal papilla cells by CCK8, EdU, and PI assay. Additionally, qPCR was also conducted to clarify the relationship between $B M P 7$ and the TGF- $\beta$ /Smad signaling pathway. A total of $1296 \mathrm{bp}$ of the BMP7 CDS region sequence was sucessfully cloned in Hu sheep, encoding a signal peptide of 431 amino acids, molecular weight was 49,316.9 Da and the isoelectric point (Pi) was 7.75. Nucleotide sequencing analysis of BMP7 revealed that Hu sheep had high homology with Bos taurus, Homo sapiens, and Canis lupus familiaris. Structure domain prediction showed that TGF- $\beta$ superfamily domain exist between 330th-431th amino acid, BMP7 protein is a secreted protein. In BMP7 up-regulated DPCs, DPCs proliferation rate and cell cycle were significantly higher than that of NC group $(p<0.05)$. Meanwhile, the expression level of Smad3, Smad4, Samd6, and TGF- $\beta 1$ in TGF- $\beta$ /Smad signaling pathway were significantly lower than that in NC group $(p<0.05)$. In $B M P 7$ down-regulated DPCs, it presented the opposite result. In conclusion, our study showed that BMP7 had a positive effect on DPCs by accelerating the proliferation and cell cycle of DPCs, and hypothesized that regulate hair follicles growth and development via TGF- $\beta /$ Smad signaling pathway. These findings may provide a synergistic target for the subsequent research of hair follicle growth and development.
\end{abstract}

Keywords: $B M P 7$; Hu sheep; dermal papilla cells; cell proliferation

\section{Introduction}

Hu sheep, as a native Chinese sheep breed, is globally well known for its rare white lambskin. Pattern type, including large waves, medium waves, small waves and straight wool [1], is the leading indicator of lambskin quality. Mechanically, the density, fineness and curvature of wool are the key factors affecting the pattern type, which are largely determined by hair follicles. Early research has proven previous observations suggesting 
that hair follicles undergo complex processes of bidirectional epithelial-mesenchymal interactions in both embryonic and birth stages, the growth of hair follicle is governed by dermal papilla cells (DPCs), which can regulate the hair follicle cycle by promoting the proliferation of epithelial cells and inducing the differentiation of epithelial stem cells [2,3]. As was previously shown in mice, fur consists of different hair types, which are characterized by the number and type of DPCs in hair follicles [2,4,5]. Besides, several signaling pathways have been confirmed to be closely related to the growth and development of hair follicles, including Wnt/ $\beta$-catenin [6] and TGF- $\beta$ /BMP [7-10].

Bone morphogenetic proteins (BMPs), are a class of functional proteins with similar structure and high conservation. As a member of the TGF- $\beta$ superfamily, it functions widely in diverse biological processes such as bone morphogenetic [11], nervous system [12], and hair follicles $[13,14]$. It is generally believed that BMPs have a suppressor function on the growth of hair follicles $[15,16]$, meanwhile, antithetical effects have also been revealed. Previous report described BMPs can promote the growth cycle of hair follicles by acting as an inhibitor of $\mathrm{Wnt} / \beta$-catenin signaling pathway [17]. Thus, BMPs may act both as a promoter or a suppressor in hair follicle growth. During the past decade, increasing members of BMPs have been identified as candidate genes in hair follicle growth and development, such as BMP2 [18,19], BMP4 [15], BMP6 [7], and BMP7 [20,21]. BMP7 has been extensively studied for its function in growth and development of reproductive system [22], pituitary [23], ovary [24], and uterus [25]. Moreover, Noramly et al. reported that BMP7 was associated with the size and spatial distribution of the feather germs [17], implying a potential role in hair follicle growth and development. In our previous research, transcriptomic profiles of the hair follicle of lambskin with large waves and small waves were obtained, and BMP7 was revealed to act as a significantly different expressed gene [26]. Considering the extensive role of $B M P 7$ in diverse biological processes, we hypothesize that BMP7 may also affect the cellular function of Hu sheep DPCs.

To evaluate this hypothesis, the BMP7 CDS region sequence of Hu sheep was cloned and performed serious of bioinformatic analysis. Then further in-depth experiments were conducted to investigate the role of $B M P 7$ by examining expression profiles, effects on cell proliferation, cell cycle via overexpression and interference of $B M P 7$ in dermal papilla cells so that we could speculate the potential function of BMP7 as a research candidate in hair follicle growth and development. Our study will provide some scientific basis for subsequent studies on the formation of wool curvature, thus laying the foundation for exploring the genetic mechanism of different pattern formation in lambskins.

\section{Materials and Methods}

\subsection{Sample Collection and Cell Culture}

Experimental lambs were supplied by Suzhou Stud Farm (Suzhou, Jiangsu Province, China). Around $1 \mathrm{~cm}^{2}$ of skin from the dorsal side of the 2-day-old Hu sheep lambs was collected and snap frozen in liquid nitrogen and then stored at $-80^{\circ} \mathrm{C}$. Total RNA was extracted using Trizol (TIANGEN, Beijing, China) per the manufacturer's instructions. First strand of cDNA was prepared using a PrimeScript ${ }^{\mathrm{TM}}$ RT Reagent Kit according to the manufacturer's instructions (Takara Bio, Beijing, China), and cDNA were stored at $-20{ }^{\circ} \mathrm{C}$.

Isolation, culture and identification of $\mathrm{Hu}$ lamb dermal papilla cells (DPCs) were followed with our laboratory methods [27]. DPCs were cultured in DMEM/F12 (Gibco, Grand Island, NY, USA) supplemented 10\% fetal bovine serum (Sigma, St. Louis, MO, USA) with $5 \% \mathrm{CO}_{2}$ at $37^{\circ} \mathrm{C}$.

All animal experimental procedures mentioned were approved by the Animal Care and Use Committee at Yangzhou University (NSFC2020-NFY-1).

\subsection{Bioinformatics Analysis of BMP7 Gene in Hu Sheep}

According to the sequences of Bos Taurus (NM_001206015.1), Homo sapiens (NM_001719.2) and Mus musculus (NM_007557.3), primers (1F, 1R) were designed to clone sheep BMP7 partial CDS. On the basis of this, $3^{\prime}$ RACE GSP inner primer (3I), $3^{\prime}$ RACE GSP outer primer 
(3O) were designed to clone $3^{\prime}$ UTR of BMP7 after the partial CDS has been obtained (Table 1). Hu sheep BMP7 full-length cDNA sequence was spliced by fragment overlapping areas, and then submitted to GenBank (No: KF925831).

Table 1. The primer for cloning of $B M P 7$.

\begin{tabular}{cclc}
\hline Gene name & Primer Name & \multicolumn{1}{c}{$\begin{array}{c}\text { Sequences } \\
\left(\mathbf{5}^{\prime} \rightarrow \mathbf{3}^{\prime}\right)\end{array}$} & Length (bp) \\
\hline \multirow{3}{*}{$B M P 7$} & $1 \mathrm{~F}$ & TAGAGCCGGCGCGATGCAC & 478 \\
& $1 \mathrm{R}$ & ACGCTACCACCACCGGGAG & 1466 \\
& $3 \mathrm{O}$ & CCTATCCCTACAAGGCCGTCT & 1095 \\
\hline
\end{tabular}

Phylogenetic tree was computed using MEGA 11 software according to the neighborjoining method based on the amino acid sequence of Hu sheep and other species such as Homo sapiens, Bos Taurus, Mus musculus, Rattus norvegicus (NM_001191856.1), Danio rerio (AF201379.1), Tegillarca granosa (JX103495.1), and Canis lupus familiaris (NM_001197052.1).

Basic chemical properties of proteins were analyzed using the ProtParam tool (ProtParam, http: / / web.expasy.org/protparam/, accessed on 22 December 2021); protein subcellular localization was analyzed using TargetP (TargetP-2.0, https: / / services.healthtech. dtu.dk/service.php?TargetP-2.0, accessed on 22 December 2021) and PSORT II Prediction (PSORT Prediction, http:/ / psort.hgc.jp/form.html, accessed on 22 December 2021); potential signal peptide cleavage sites prediction were performed using SignalP4.1 (SignalP4.1, https:/ / services.healthtech.dtu.dk/service.php?SignalP-4.1, accessed on 22 December 2021); glycosylation sites were analyzed using NetOGlyc4.0 (NetOGlyc4.0, https: / / services.healthtech.dtu.dk/service.php?NetOGlyc-4.0, accessed on 22 December 2021); the phosphorylation sites of amino acids were predicted using NetPhos3.1 (NetPhos-3.1, https: / / services.healthtech.dtu.dk/service.php?NetPhos-3.1, accessed on 22 December 2021); conserved domains of the amino acid sequence were predicted using Smart (SMART, http:/ / smart.embl-heidelberg.de/, accessed on 22 December 2021); the hydrophilicity of amino acid sequences was analyzed using ProtScale (ProtScale, http:/ / web.expasy. org/protscale/, accessed on 22 December 2021); protein secondary structure was predicted using GOR IV (GOR IV SECONDARY STRUCTURE PREDICTION METHOD, http:/ / npsa-pbil.ibcp.fr / cgi-bin/npsa_automat.pl?page=npsa_gor4.html, accessed on 22 December 2021); transmembrane analysis of protein sequences was performed using TMHMM-2.0 (TMHMM-2.0, https:/ / services.healthtech.dtu.dk/service.php?TMHMM-2.0, accessed on 22 December 2021).

\subsection{Over Expression and Inhibition of BMP7}

The CDS sequence of BMP7 gene was amplified from the cDNA by PCR using the gene-specific primers designed from the $\mathrm{Hu}$ sheep BMP7 gene. The restriction sites of Xho I and Kpn I (underlined in the sequences below) were introduced into the forward primer (5'-cCCTCGAGATGCACATGCGCTCGCTAC-3') and the reverse primer $\left(5^{\prime}\right.$ ggGGTACCCTAGTGGCAGCCACAAGCCC $-3^{\prime}$ ), respectively. The PCR product was gelpurified, sequenced and ligated into pEX-1 vector using DNA Ligation Kit (Takara Bio, Beijing, China).

Small interfering RNA against BMP7 (siRNA-BMP7) were designed and synthesized by GenePharma (Shanghai, China), details were provided in Table 2.

pEX-1-BMP7 and siRNA-BMP7 were transfected into DPCs respectively using jetPRIME transfection reagent (Polyplus-transfection, Illkirch-Graffenstaden, France). DPCs were collected for following studies at $24 \mathrm{~h}$ after transfections, including Negative control (NC). 
Table 2. The sequence information of siRNA-BMP7.

\begin{tabular}{cc}
\hline Name & Sequences $\left(\mathbf{5}^{\prime} \rightarrow \mathbf{3}^{\prime}\right)$ \\
\hline siRNA-124 & $\begin{array}{c}\text { sense: GCACUCGAGCUUCAUCCAUTT } \\
\text { antisense: AUGGAUGAAGCUCGAGUGCTT } \\
\text { sense: CCACCCGGGAGUUCCGGUUUTT } \\
\text { siRNA-469 }\end{array}$ \\
siRNA-55e: AAACCGGAACUCCCGGUGGTT \\
sense: CCGGGAACACUUCCACAAUTT \\
antisense: AUUGUGGAAGUGUUCCCGGTT
\end{tabular}

\subsection{Cell Proliferation and Cycle Assay}

The DPCs were transferred to 96-well plates before cell proliferation assay, $10 \mu \mathrm{L}$ Cell Counting Kit-8 (CCK-8) solution was added to each well at 0, 24, 48 and $72 \mathrm{~h}$ after transfections. The OD value was measured at $450 \mathrm{~nm}$ with a Tecan Infinite F200/M200 microplate reader (Tecan, Männedorf, Switzerland) after $2 \mathrm{~h}$. The proliferation of DPCs was also detected at $24 \mathrm{~h}$ after transfections using the Cell-Light EdU Apollo567 In Vitro Kit (RiboBio, Guangzhou, China) according to the manufacturer's instructions.

The cell cycle of DPCs was also detected at $24 \mathrm{~h}$ after transfections using Cell Cycle and Apoptosis Analysis Kit (Beyotime, Shanghai, China) according to the manufacturer's instructions by flow cytometry.

\section{5. $q R T-P C R$}

After overexpression and disruption of $B M P 7$ in dermal papilla cells, several key genes (Smad1, Smad3, Smad4, Smad5, Smad6, and TGF- $\beta 1$ ) in the TGF- $\beta /$ Smad signaling pathway were selected for qRT-PCR.Total RNA was extracted and first strand of cDNA was prepared. Based on assembled sequences of studied genes in GenBank, primers were designed using the Primer Premier v5.0 software (Table 3). All primers were synthesized by Sango Biotechnology Co., Ltd. (Beijing, China). Taking Lu et al. s' research as a reference [28], house-keeping gene $(G A P D H)$ was used as an internal control to normalize the threshold cycle (CT) values. The $2^{-\Delta \Delta C T}$ method was used to process real-time PCR results [29].

Table 3. The primer information of qRT-PCR.

\begin{tabular}{|c|c|c|c|c|}
\hline $\begin{array}{l}\text { Gene } \\
\text { Name }\end{array}$ & $\begin{array}{c}\text { Sequences }\left(5^{\prime} \rightarrow 3^{\prime}\right) \\
\text { (F: Forward R: Reverse) }\end{array}$ & $\begin{array}{l}\text { Length } \\
\text { (bp) }\end{array}$ & $\begin{array}{l}\mathrm{Tm} \\
\left({ }^{\circ} \mathrm{C}\right)\end{array}$ & Accession No. \\
\hline$B M P 7$ & $\begin{array}{l}\text { F: TGAGTTCCGCATTTACAAGG } \\
\text { R: GTGGCTGTGATGTCAAAAAC }\end{array}$ & 177 & 60 & KF925831.1 \\
\hline Smad1 & $\begin{array}{l}\text { F: GAAAGCCCCGTTCTTCCTCC } \\
\text { R: GTTGGGCTGCTGGAAAGAAT }\end{array}$ & 150 & 60 & AY035385.1 \\
\hline Smad3 & $\begin{array}{l}\text { F: GGACGACTACAGCCATTCCA } \\
\text { R: ATTCGGGGAGAGGTTTGGAG }\end{array}$ & 172 & 60 & AF508024.1 \\
\hline Smad4 & $\begin{array}{c}\text { F: CTTCAGGTGGCTGGTCGG } \\
\text { R: TCCAGGTGATACAACTCGTTCA }\end{array}$ & 177 & 60 & NM_001267886.1 \\
\hline Smad5 & $\begin{array}{l}\text { F: CCAGTATATCCAGCAGAGATGTT } \\
\text { R: AAGCTTCCCCAACACGATTG }\end{array}$ & 102 & 60 & AF508027.1 \\
\hline Smad6 & $\begin{array}{l}\text { F: CTGCTCGGACGCCTCTTC } \\
\text { R: GGGTGGCGGTGATTCTGG }\end{array}$ & 105 & 60 & XM_004010255.5 \\
\hline TGF- $\beta 1$ & $\begin{array}{l}\text { F: GGTGGAATACGGCAACAAAATC } \\
\text { R: TGCTGCTCCACTTTTAACTTGA }\end{array}$ & 162 & 60 & NM_001009400.2 \\
\hline GAPDH & $\begin{array}{l}\text { F: GTCGGAGTGAACGGATTTGG } \\
\text { R: CATTGATGACGAGCTTCCCG }\end{array}$ & 196 & 60 & NM_001190390.1 \\
\hline
\end{tabular}

Note: Bone morphogenetic protein 7 (BMP7), SMAD family member 1 (Smad1), SMAD family member 3 (Smad3), SMAD family member 4 (Smad4), SMAD family member 5 (Smad5), SMAD family member 6 (Smad6), transforming growth factor beta 1 (TGF- $\beta 1)$, glyceraldehyde-3-phosphate dehydrogenase (GAPDH). 


\subsection{Western Bolt}

After the DPCs were transfected for $36 \mathrm{~h}$, the protein of DPCs were disposed using RIPA Lysis Buffer (Beyotime, Shanghai, China), protein concentrations were measured using Enhanced BCA Protein Assay Kit (Beyotime, Shanghai, China). The proteins were separated by SDS-PAGE, transferred to polyvinylidene difluoride (PVDF) membranes, and probed with 1:500 rabbit anti-BMP7 (Huabio, Hangzhou, China) and 1:2500 rabbit anti-GAPDH (Proteintech Group, Rosemont, IL, USA) antibodies, and then with 1:3000 goat anti-rabbit IgG HRG antibodies (ABclonal, Wuhan, China). Signals were detected with the enhanced chemiluminescence ECL Western Blot kit (Biosharp, Hefei, China). All experimental producers were performed per the manufacturer's instructions. Protein was detected and analyzed by the ChemiDoc ${ }^{\mathrm{TM}}$ Analysis System (Bio-Rad, Hercules, CA, USA).

\subsection{Statistical Analysis}

All mentioned experiments were performed in triplicate to ensure the reliability of our study. The $2^{-\Delta \Delta C T}$ method was used to process real-time PCR results [29]. Statistical analyses were carried out using SPSS 18.0 software (IBM, Armonk, NY, USA). Independent sample t-test and one-way analysis of variance (ANOVA) were used to perform variance analysis and significance test. All experimental data are presented as mean \pm standard error of the mean (SEM). A probability of $p \leq 0.05$ was considered statistically significant, and a probability of $p \leq 0.01$ was considered to be extremely statistically significant.

\section{Results}

\subsection{Cloning Hu Sheep BMP7 Full Length cDNA Sequence}

The 478 bp CDS sequence was successfully cloned by $1 \mathrm{~F}$ and $1 \mathrm{R}$ amplification on cDNA. 3' RACE was used to clone 1095 bp sequence. A total of 1296 bp CDS region sequence was obtained after spliced these two sequences according to overlapping sequence, and submitted to GenBank (No: KF925831).

\subsection{Bioinformatics Analysis of BMP7 Gene in Hu Sheep}

Similarity analysis was computed on nucleotide and amino acid sequence of $\mathrm{Hu}$ sheep's BMP7 CDS region and Homo sapiens, Bos Taurus, Mus musculus, Rattus norvegicus, Danio rerio, Tegillarca granosa, and Canis lupus familiaris (Table 4). The results of nucleotide similarity analysis showed the highest homology existed between Hu sheep and Bos Taurus $(98.5 \%)$, followed by Homo sapiens $(92.7 \%)$, the results of amino acid similarity analysis showed the highest homology existed between Hu sheep and Bos Taurus (99.4\%), followed by Mus musculus, and Rattus norvegicus (98.9\%), phylogenetic tree was also constructed based on amino acid sequence (Figure 1A), which also suggested a high similarity between these species.

Table 4. Similarity analysis of nucleotide and amino acid of BMP7.

\begin{tabular}{cccc}
\hline Species & Accession No. & Nucleotide (\%) & Amino Acid (\%) \\
\hline Homo sapiens & NM_001719.2 & $92.7 \%$ & $98.7 \%$ \\
Bos taurus & NM_001206015.1 & $98.5 \%$ & $99.4 \%$ \\
Mus musculus & NM_007557.3 & $89 \%$ & $98.9 \%$ \\
Rattus norvegicus & NM_001191856.1 & $88 \%$ & $98.9 \%$ \\
Danio rerio & AF201379.1 & $68 \%$ & $88.4 \%$ \\
Tegillarca granosa & JX103495.1 & $48.7 \%$ & $79.5 \%$ \\
Canis lupus familiaris & NM_001197052.1 & $92.1 \%$ & $98.5 \%$ \\
\hline
\end{tabular}


A

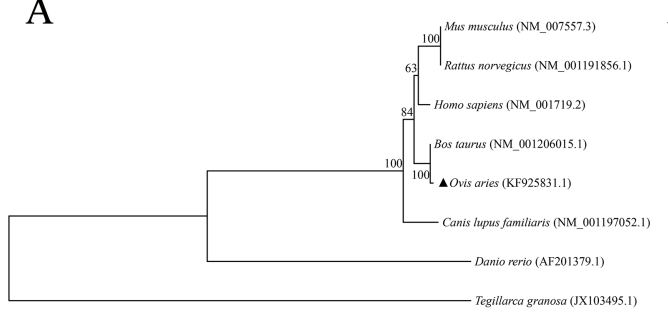

0.05

$\mathrm{C}$

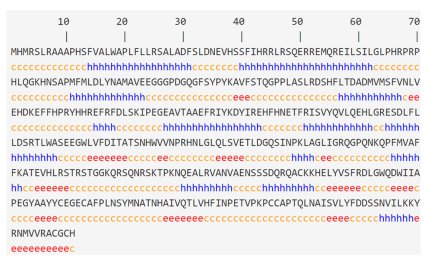

E

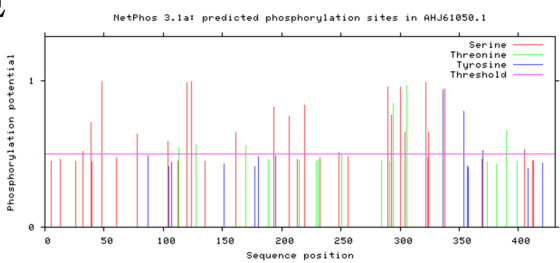

F

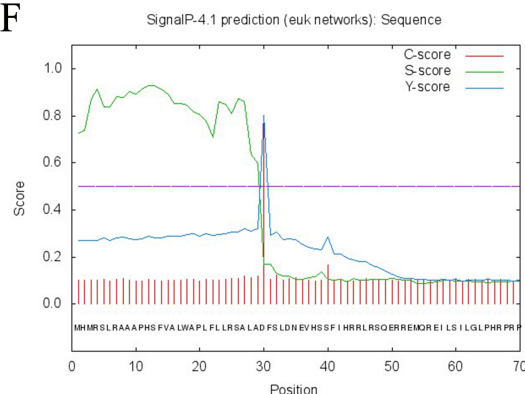

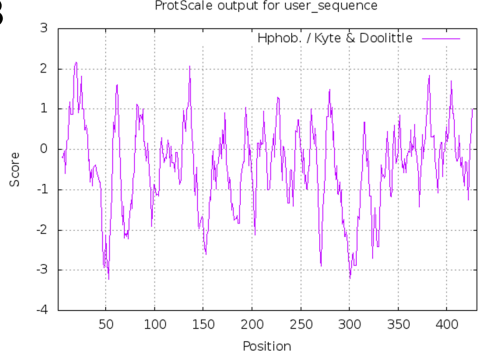

D

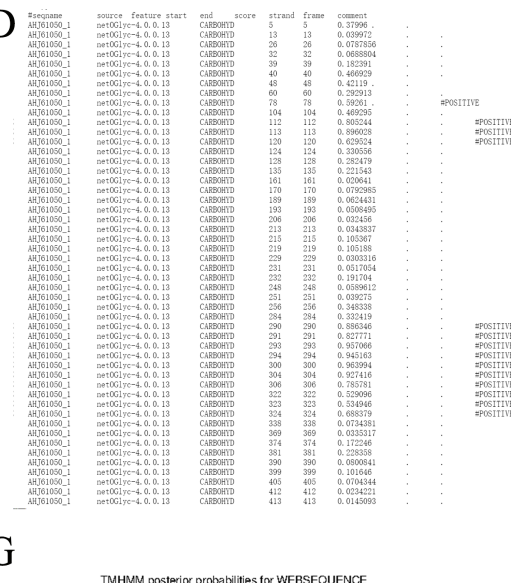

G

TMHMM posterior probabalitites for WEBSEQUENCE

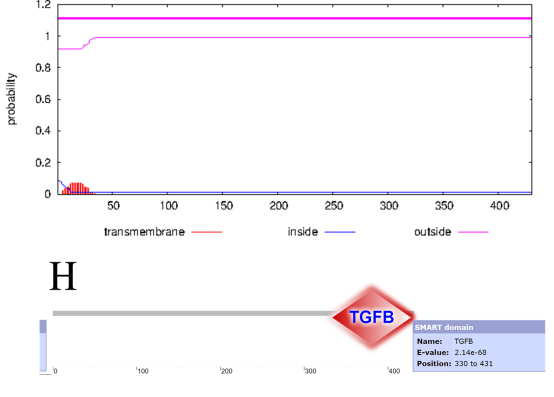

Figure 1. Bioinformatics analysis of $B M P 7$ gene in Hu sheep. (A) Analysis of phylogenetic tree for $B M P 7$ gene. (B) The hydropathy profile of BMP7 sheep amino acid. Note: The horizontal scale indicated the number of amino acid residues and the vertical one was the relative hydropathy scale. Points above the zero horizontal line corresponded to hydrophobic region, and points below the line were hydrophilic. (C) BMP7 protein secondary structure prediction. (D) BMP7 glycosyl site analyses. (E) BMP7 phosphorylation site analyses. (F) BMP7 signal peptide prediction. (G) BMP7 protein transmembrane regional analyses. (H) BMP7 conservative structure domain analyses.

As is mentioned above, serious of bioinformatics analysis were performed. The CDS region of Hu sheep's BMP7 was $1296 \mathrm{bp}$, encoding a signal peptide of 431 amino acids, molecular weight was 49,316.9Da and the isoelectric point (Pi) was 7.75. Most of the amino acid sequence contained hydrophilic residues, which indicated that $\mathrm{Hu}$ sheep's BMP7 protein was water-soluble (Figure 1B). Result of subcellular localization showed that $B M P 7$ protein was a secreted protein (Table 5). Secondary structure prediction of components of BMP7 protein showed that $\alpha$-helix (h) accounted for $34.11 \%$, $\beta$-sheet (e) accounted for $14.62 \%$, and random coils (c) accounted for 51.28\% (Figure 1C). Structure prediction revealed that BMP7 protein had 14 potential glycosylation sites (Figure 1D), 30 potential phosphorylation sites (Figure 1E), a signal peptide cleavage site between 29th-30th (Figure 1F), and no transmembrane helices structure in BMP7 protein (Figure 1G). Further in-depth structure domain prediction showed that TGF- $\beta$ superfamily domain existed between 330th-431th amino acid (Figure 1H). Functional analysis of BMP7 protein 
revealed that $B M P 7$ protein was major enriched in biosynthesis of cofactors, transport and binding, and translation, details were shown in Table 6.

Table 5. Subcellular localization of BMP7.

\begin{tabular}{ccccc}
\hline Name & Length & mTP & SP & Other \\
\hline AHJ61050.1 & 431 & 0.327 & 0.858 & 0.015 \\
\hline
\end{tabular}

Table 6. The function analysis of $B M P 7$ protein.

\begin{tabular}{cc}
\hline Functional Category & Odds \\
\hline Amino acid biosynthesis & 0.500 \\
Biosynthesis of cofactors & 2.917 \\
Cell envelope & 0.541 \\
Cellular processes & 0.411 \\
Central intermediary metabolism & 0.762 \\
Energy metabolism & 0.389 \\
Purines and pyrimidines & 1.308 \\
Fatty acid metabolism & 1.362 \\
Replication and transcription & 0.211 \\
Regulatory functions & 0.075 \\
Translation & 1.614 \\
Transport and binding & 1.885 \\
\hline
\end{tabular}

\subsection{BMP7 Promoted Proliferation of Hu Sheep DPCs}

To estimate the role of $B M P 7$ in DPCs, we designed over-expression vector (pEX1-BMP7) and siRNA (siRNA-BMP7s) specifically targeted BMP7. First, we verified the transfection effects of $\mathrm{pEX}-1-B M P 7$ and siRNA-BMP7s, at $24 \mathrm{~h}$ post transfection, both $\mathrm{pEX}$ 1-BMP7-transfected clones and siRNA-BMP7s transfected clones showed clearly visible fluorescence signal (Figures 2A and 3A), the results of RT-qPCR (Figure 2B) and Western bolt (Figure 2C) showed that $\mathrm{pEX}-1-B M P 7$ can up-regulate the expression of $B M P 7$ at $\mathrm{mRNA}$ and protein level. Regarding siRNAs, detectable interfering mRNA levels of $B M P 7$ were observed after the transfection of all three siRNA-BMP7s (Figure 3B), within which, the transfection effects of siRNA-124 were higher compared with other control-transfected siRNAs at statistically significant level $(p<0.05)$, and lowest level of BMP7 mRNA expression were obtained with siRNA-124 at a concentration of $50 \mathrm{nM}$ (Figure 3C). Furthermore, the results of Western bolt (Figure 3D) showed that the transfection of siRNA-124 at a concentration of $50 \mathrm{nM}$ can down-regulate the expression of $B M P 7$ protein. Collectively, our results showed that BMP7 could be successfully over/down expressed in DPCs by transfection.

To determine whether BMP7 affects DPCs proliferation, we quantified cell proliferation and cycle of DPCs. As measured by CCK-8 assay, the proliferation rate was significantly higher in $\mathrm{pEX}-1-B M P 7-$ transfected DPCs than that of negative control ( $\mathrm{pEX}-1$ transfected) DPCs (Figure 4A), as expected, lower proliferation rate was revealed in siRNA124-transfected DPCs than that of non-targeting siRNA-transfected DPCs, especially at 24 and $72 \mathrm{~h}$ post transfection (Figure 5A). Results were similar in Edu assay, the transfection of pEX-1-BMP7 can significantly promote the proliferation of DPCs (Figure 4B,C), and cell proliferation can be inhibited by the downregulation of BMP7 in DPCs (Figure 5B,C). Next, the cell cycle was analyzed by flow cytometry. The results showed that proportion of cells in S phase were notably higher in pEX-1-BMP7-transfected DPCs compared to NC (Figure 6), and lower proportion of cells in S phase were observed in siRNA-124-transfected DPCs compared to NC (Figure 7). 
A
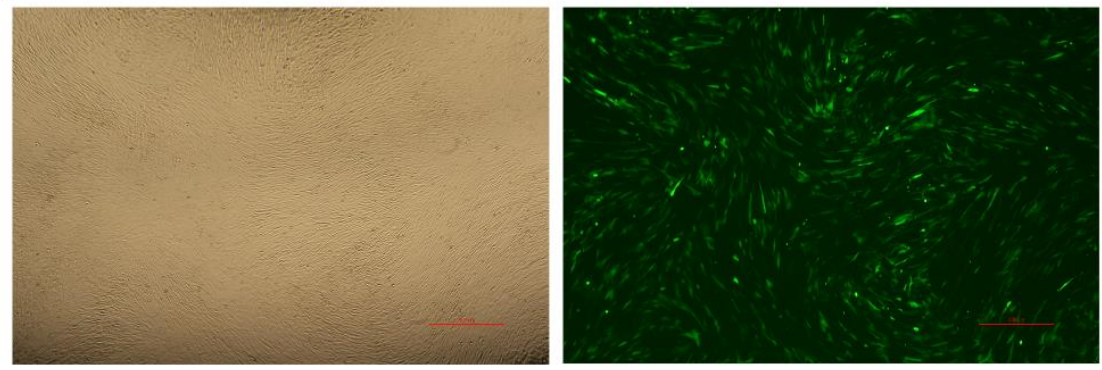

B

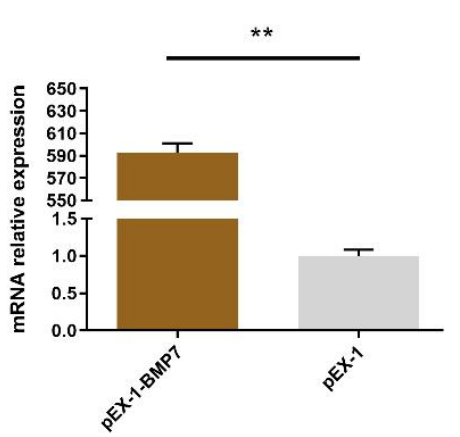

C

BMP7

GAPDH

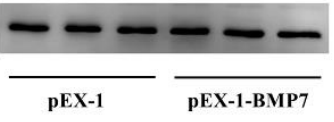

Figure 2. Validation of $B M P 7$ transfection efficiency. (A) Transfection efficiency by green fluorescent protein. (B) The mRNA expression level of BMP7. Note: “*** represents an extremely significant difference $(* * p 0.01)$. (C) The protein level of BMP7.

A

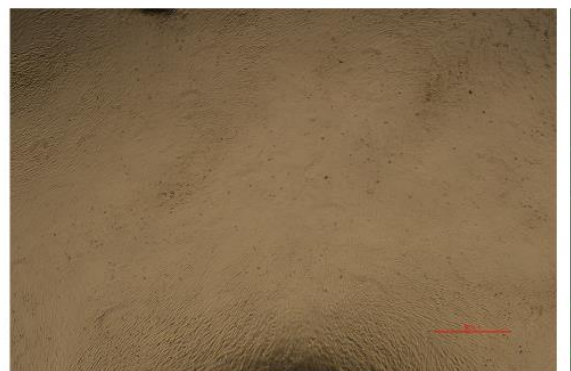

B

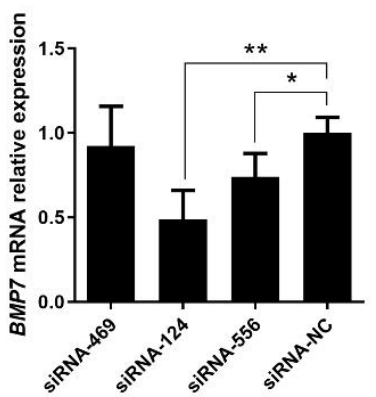

C

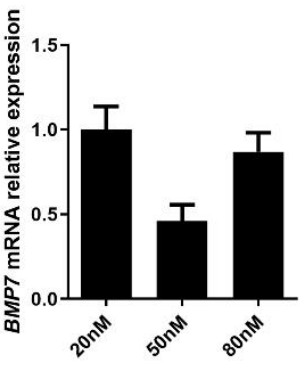

D

BMP7

GAPDH

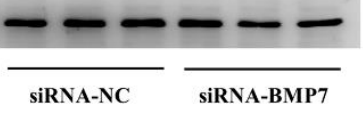

Figure 3. The mRNA and protein level of BMP7 in dermal papilla cells transfected with siRNA-BMP7. (A) Transfection efficiency by green fluorescent protein. $(\mathbf{B}, \mathbf{C})$ The mRNA expression level of BMP7. (D) The protein level of BMP7. "** represents a significant difference $(p<0.05)$ and "**" represents an extremely significant difference $(p<0.01)$. 
A

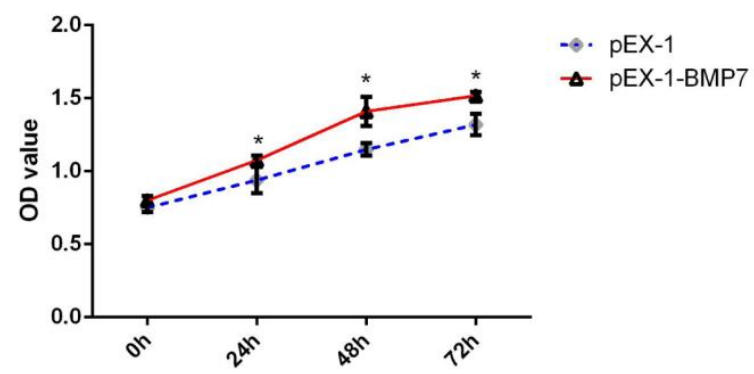

B

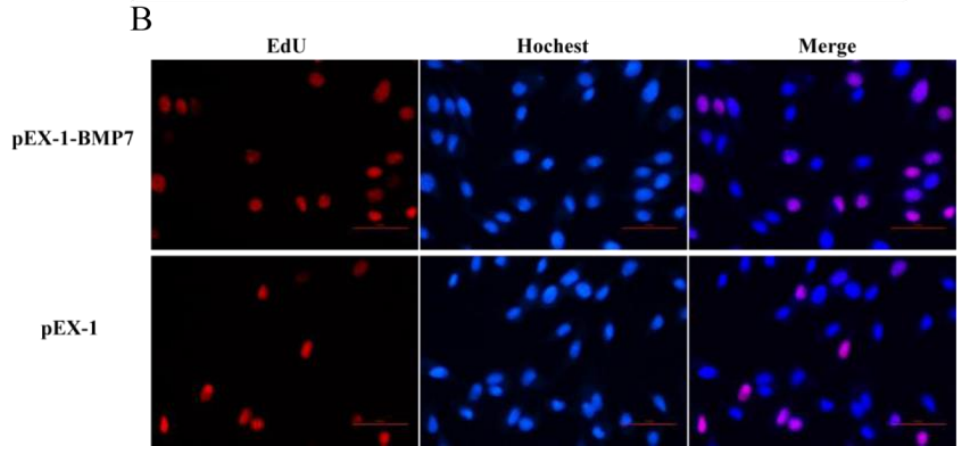

$\mathrm{C}$

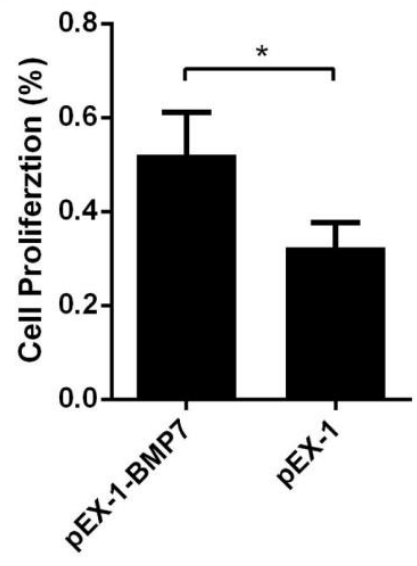

Figure 4. The effects of $B M P 7$ over-expression on dermal papilla cell proliferation of Hu sheep. (A) Detection of dermal papilla cell activity by CCK-8 after over-expression of BMP7 gene. (B) Detection of dermal papilla cell proliferation by EdU after over-expression of $B M P 7$ gene. $(\mathbf{C})$ The rate of proliferating cells. "*" represents a significant difference $(p<0.05)$.

A
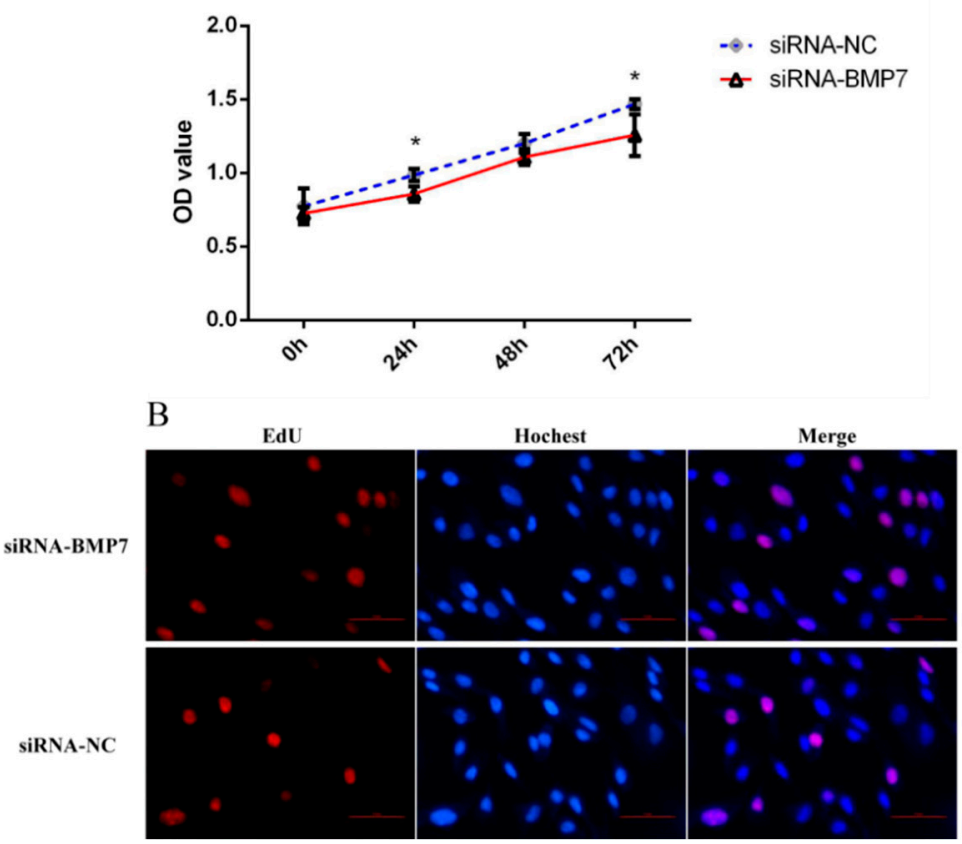

C

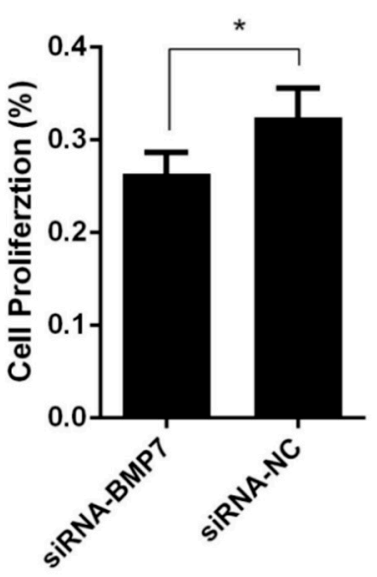

Figure 5. The effects of $B M P 7$ down-regulation on dermal papilla cell proliferation of Hu sheep. (A) Detection of dermal papilla cell activity by CCK-8 after BMP7 down-regulation. (B) Detection of dermal papilla cell proliferation by EdU after BMP7 down-regulation. (C) The rate of proliferating cell. "*”" represents a significant difference $(p<0.05)$. 
A
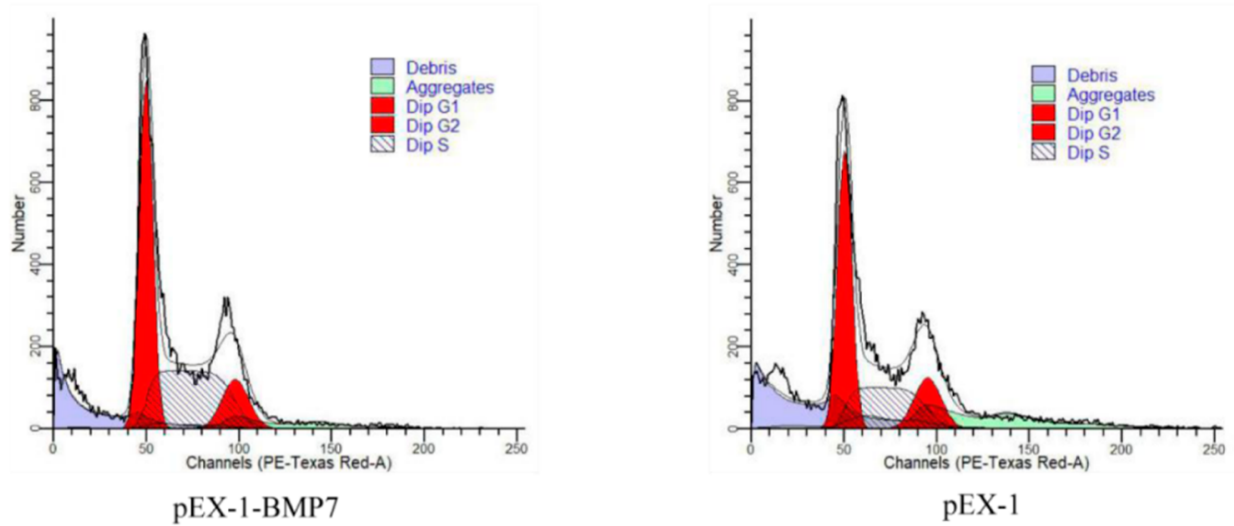

B

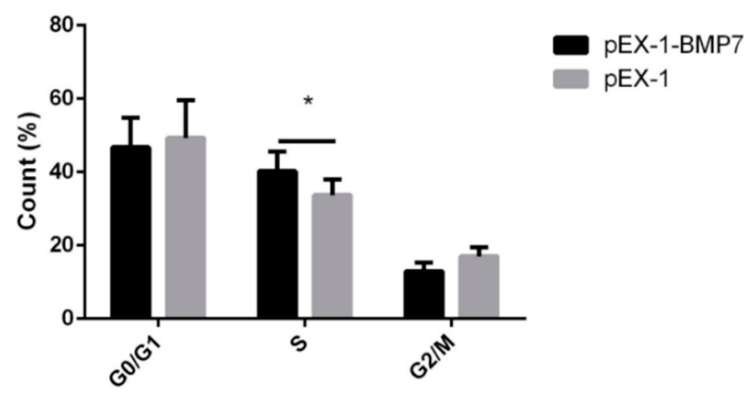

Figure 6. The effects of dermal papilla cell cycle after BMP7 over-expression. (A) The dermal papilla cell cycle after BMP7 over-expression. (B) The rate of different periods of the cell cycle. "**" represents a significant difference $(p<0.05)$.

A

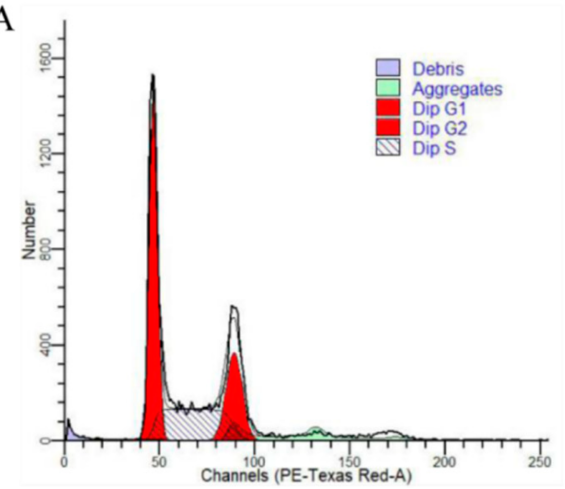

siRNA-BMP7

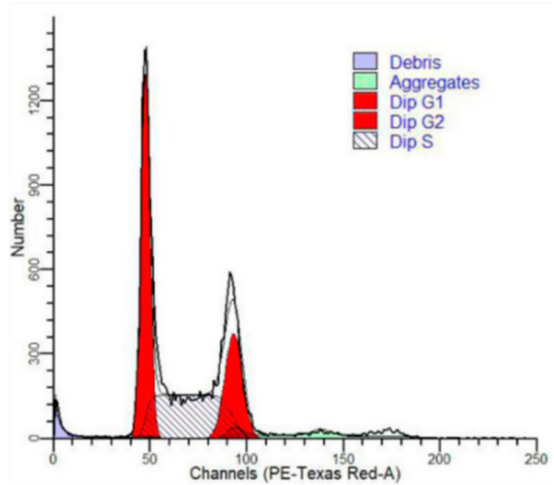

siRNA-NC

B

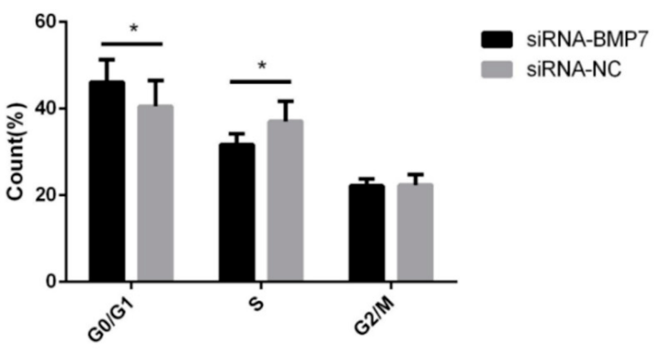

Figure 7. The effects of dermal papilla cell cycle after $B M P 7$ down-regulation. (A) The dermal papilla cell cycle after BMP7 down-regulation. (B) The rate of different periods of the cell cycle. "**" represents a significant difference $(p<0.05)$. 


\subsection{BMP7 Regulated Key Genes in TGF- $\beta /$ Smad Signaling Pathway}

Given that TGF- $\beta$ /Smad signaling pathway was involved in diverse cellular biological processes and the crucial role of BMP7 in TGF- $\beta$ /Smad signaling pathway, we evaluated the mRNA expression levels of the key genes in the TGF- $\beta$ /Smad signaling pathway (Smad1, Smad3, Smad4, Smad5, Smad6, and TGF- $\beta 1$ ) after transfection using qRT-PCR. As shown in Figure 8A, the expression level of Smad3, Smad4, Smad6, and TGF- $\beta 1$ were significant lower in pEX-1-BMP7-transfected DPCs compared to NC $(p<0.05)$, whereas the expression levels of Smad1 and Smad5 showed non-significant difference. After down-regulating BMP7 in DPCs (Figure 8B), the expression levels of Smad3, Smad4, Smad5, and TGF- $\beta 1$ were significantly up regulated $(p<0.05)$, non-significant difference was noted in the expression levels of Smad1 and Smad6. Besides, In BMP7 up-regulated DPCs and BMP7 down-regulated DPCs groups, notably, Smad3, Smad4, Smad6, and TGF- $\beta 1$ genes showed the opposite trends after transfection.

A

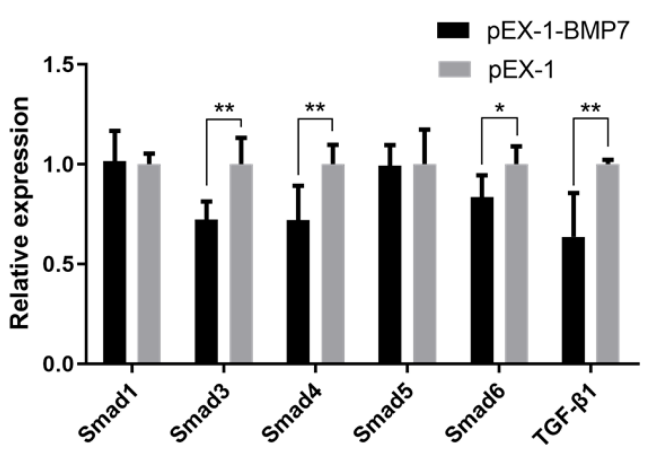

B

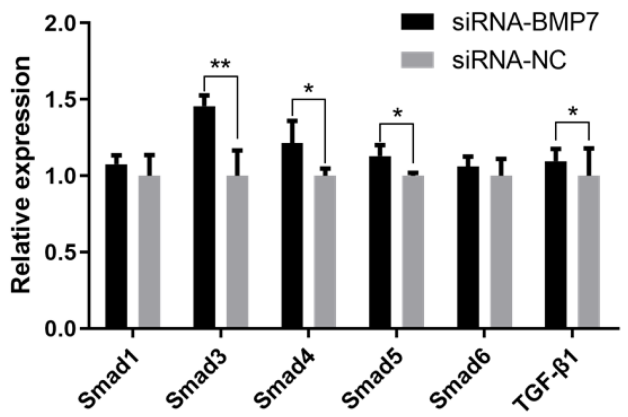

Figure 8. The gene expression of the TGF- $\beta /$ Smad signaling pathway. (A) The effects of BMP7 over-expression on the gene expression of the TGF- $\beta /$ Smad signaling pathway. (B) The effects of $B M P 7$ interference on the gene expression of the TGF- $\beta /$ Smad signaling pathway. "**" represents a significant difference $(p<0.05)$ and "***" represents an extremely significant difference $(p<0.01)$.

\section{Discussion}

The quality of lambskin in Hu sheep is influenced by the pattern type, which is closely associated with the wool curvature. Previous research has found that it can lead to pressure on one side of the wool fiber, ultimately giving rise to wool curvature, when the cell proliferation was asymmetric on both sides of hair follicle. To a certain degree, the greater the asymmetry of cell proliferation, the greater the pressure, and the more obvious the bending degree of wool fiber. Therefore, the asymmetry of cell proliferation in hair follicles was considered to be a significant factor affecting wool curvature [30]. Multiple growth factors and intercellular signaling molecules, expressed and secreted by dermal papilla cells, had an impact on the formation of hair follicle symmetry/asymmetry axis, including Wnt, TGF- $\beta$ /BMP, Notch, Shh, FGFs and other regulatory factors. Kwack et al. reported that these factors regulated the proliferation and differentiation of cells in hair follicles by acting on neighboring cells through paracrine pathway [31]. In addition, Nissimov et al. proposed the hypothesis of "Multiple papillary centers" (MPC), which explained the cytological mechanism of hair bending [32]. MPC, referred to two or more dermal papilla regions, and the hypothesis shown that these dermal papillae can induce the growth rate of different cortical cells in their adjacent areas independently, leading to the difference of cell growth rate and eventually resulting in hair bending. In view of the effects of dermal papilla cells on the proliferation and differentiation of various cells in hair follicles, even on hair bending, we carried out experiments with dermal papilla cells as the core.

As is described in our preliminary transcriptomic data, $B M P 7$, one of the most biologically active proteins in the BMP family, was identified as significant differently expressed gene between the large waves and small waves of the hair follicle in Hu sheep lambskin [26]. 
Existing studies have proved that BMP family can regulate the proliferation, apoptosis and differentiation of cells in hair follicles and play respective roles in different cells, finally affecting the growth and development of hair follicles $[13,33]$. It has been found that overexpression of $B M P 2$ increased the expression of differentiation marker factors and decreased the expression of proliferation marker factors in hair follicle stem cells, which promoted the differentiation process of hair follicle stem cells $[18,19]$. Wu et al. reported that BMP6 regulated the transition of hair follicle from telogen phase to anagen phase through acting as an inhibitor of hair follicle stem cell activation [7], and the similar inhibitory effect was also found in BMP4 on hair follicle growth and development $[15,16,34]$. It is worth mentioning that the expression levels of BMP4 and BMP6 would be elevated while hair follicles were activated and began to grow [35,36], which appeared to be contrary to the previously discovered theory that BMPs signal was an inhibitory signal $[7,15,16,34]$. Nevertheless, some researchers considered it was a negative feedback loop after in-depth understanding $[35,36]$. Although no evidence that BMP7 can directly regulate wool curvature have been reported yet, several findings suggested that $B M P 7$ played a direct role in the growth and development of hair follicles [37,38]. A more general involvement of BMP7 in the development of hair follicle and thereby epithelial-mesenchymal interactions was indicated by its strong expression in developing hair follicles, and the concomitant morphological deformation of structure [38]. Apparently, BMP7 could participate in the formation of several traits in wool, but the specific regulatory mechanism is unclear. Considering the critical role of dermal papilla cells, and the participation of BMP7 in hair follicle growth and development, we can hypothesis that BMP7 may also play a role in the hair follicle growth and development by regulating the cellular process of DPCs, hence our study was designed and tried to provide an insight into the cellular function of BMP7 in DPCs and further clarify the role of $B M P 7$ in hair follicle growth and development.

In our present study, in BMP7-upregulated DPCs, proliferation rate was promoted and the cell cycle was driven more rapidly. Meanwhile, proliferation rate and cell cycle was inhibited by down regulation of $B M P 7$. In contrast to most previous findings, which had suggested suppressor function of BMPs in hair follicle growth and development $[15,16]$, our study investigated that $B M P 7$ can promote cell proliferation and driven cell cycle of $\mathrm{Hu}$ sheep DPCs. In order to explain these differences, one should take into consideration that the negative feedback regulation and increased expression of BMP4 in the early stage of hair follicle growth $[35,36]$, hence we assume that negative feedback regulation may also exist between BMP7 and hair follicle growth. By contrast, it is worth noting that our results are closer to Adly et al. s' research [21]. As shown in their study on the relationship between $B M P 7$ protein and human hair follicle cycling, BMP7 was found highly expressed during the growth phase of hair follicle, including outer root sheath, inner root sheath, fur layer cells, dermal papilla, and connective tissue sheath. Additionally, high expression level of BMP7 was also detected in these cells during the proliferation phase of hair follicle cells. As hair follicle cells proliferate to the catagen and telogen phase during which BMP7 was always found expressed, then BMP7 was no longer expressed in most hair follicle cells, and the expression of $B M P 7$ showed a corresponding decrease in dermal papilla. Combing our data with predecessors' reports, it is reasonable to believe that BMP7 can participate in the growth and development of hair follicles of $\mathrm{Hu}$ sheep through promoting cell proliferation and driving cell cycle of DPCs, and may even affect the curvature of wool and the formation of lambskin pattern.

Known signaling pathways such as Wnt/ $\beta$-catenin, EDA/EDAR, TGF- $\beta /$ Smad and Notch interrelate and interact with each other to form a complex network to regulate the occurrence and cycle of hair follicles, and then affect hair traits [39,40]. The mechanisms associated with TGF- $\beta$ /Smad signaling pathway related to BMPs have been partially explained in recent studies. It has been found that decorin (DCN) had a certain impact on the morphogenesis and periodic changes of hair follicles by way of promoting TGF- $\beta$ and BMP signal transduction pathway [9]. Meanwhile, activation of Smad4 in TGF- $\beta$ /Smad signaling pathway can lead to hair fiber keratinization [41], and activate expression of 
downstream target genes, further affecting proliferation and differentiation of epithelial cells [42]. In addition, Song et al. reported that the low expression of BMP2 and BMPR-IA in the telogen phase supported their inhibitory effect on the growth of hair follicle cells in yak [8]. BMP7 was reported to act on TGF- $\beta /$ Smad signaling pathway to realize signal transduction via binding to the BMPs receptors (BMPRs) on the cell membrane [43]. To evaluate the connection between BMP7 and TGF- $\beta$ /Smad signaling pathway in hair follicle growth, we performed the qPCR to measure the mRNA expression level of several key candidate genes in TGF- $\beta$ /Smad signaling pathway (Smad1, Smad3, Smad4, Smad5, Smad6, and TGF- $\beta 1$ ). In BMP7 up/down-regulated DPCs, the results showed that Smad3, Smad4, Smad6, and TGF- $\beta 1$ genes showed the opposite pattern, however, non-significant difference was noted in the expression level of Smad1. In light of the aforementioned observations, we could speculate that $B M P 7$ may regulate hair follicle growth via influencing the signal transduction pathway or key candidate genes of TGF- $\beta$ /Smad signaling pathway. Of course, further research is needed to confirm our speculation. In conclusion, our study shown that overexpression of BMP7 resulted in accelerating the proliferation and cell cycle of DPCs, which can also be inhibited by downregulation of BMP7. Furthermore, qPCR results suggested that BMP7 may regulate hair follicle growth via TGF- $\beta / \mathrm{Smad}$ signaling pathway. Given the important roles of BMPs, which interact with the TGF$\beta /$ Smad signaling pathway, our novel findings suggest a new direction for research of hair follicle growth and development, and can provide molecular basic for the breeding of Hu sheep.

Author Contributions: X.L. and W.S. designed this study; Y.L. and X.L. performed the experiments and wrote the manuscript; S.W., X.C., Z.Y. contributed to materials and data collection; T.G., J.M.M. and A.H. revised the final manuscript. All authors have read and agreed to the published version of the manuscript.

Funding: This research was funded by National Natural Science Foundation of China (31872333, 32172689), National Natural Science Foundation of China-CGIAR (32061143036), Key Research and Development Plan (modern agriculture) in Jiangsu Province (BE2018354), Major New Varieties of Agricultural Projects in Jiangsu Province (PZCZ201739), Jiangsu Agricultural Science and Technology Innovation Fund (CX (18)2003).

Institutional Review Board Statement: Sample collection protocol was approved by the Animal Care and Use Committee at Yangzhou University (No: NSFC2020-NFY-1). All animal experimental procedures were implemented in strict accordance with the guidelines for the Animal Care and Use Committee at Yangzhou University to minimize the suffering of animals.

Informed Consent Statement: Not applicable.

Data Availability Statement: The raw data supporting the conclusions of this article will be made available by the authors, without undue reservation.

Acknowledgments: The authors would like to acknowledge to Suzhou Stud Farm in Jiangsu Province, China for providing sheep samples.

Conflicts of Interest: The authors declare no conflict of interest.

\section{References}

1. Lv, X.; Chen, W.; Sun, W.; Hussain, Z.; Wang, S.; Wang, J. Analysis of lncRNAs Expression Profiles in Hair Follicle of Hu Sheep Lambskin. Animals 2020, 10, 1035. [CrossRef]

2. Chi, W.; Wu, E.; Morgan, B.A. Dermal papilla cell number specifies hair size, shape and cycling and its reduction causes follicular decline. Development 2013, 140, 1676-1683. [CrossRef]

3. Morgan, B.A. The dermal papilla: An instructive niche for epithelial stem and progenitor cells in development and regeneration of the hair follicle. Cold Spring Harb. Perspect Med. 2014, 4, a015180. [CrossRef] [PubMed]

4. Driskell, R.R.; Giangreco, A.; Jensen, K.B.; Mulder, K.W.; Watt, F.M. Sox2-positive dermal papilla cells specify hair follicle type in mammalian epidermis. Development 2009, 136, 2815-2823. [CrossRef] [PubMed]

5. Rompolas, P.; Deschene, E.R.; Zito, G.; Gonzalez, D.G.; Saotome, I.; Haberman, A.M.; Greco, V. Live imaging of stem cell and progeny behaviour in physiological hair-follicle regeneration. Nature 2012, 487, 496-499. [CrossRef] 
6. Wang, J.; Cui, K.; Yang, Z.; Li, T.; Hua, G.; Han, D.; Yao, Y.; Chen, J.; Deng, X.; Yang, X.; et al. Transcriptome Analysis of Improved Wool Production in Skin-Specific Transgenic Sheep Overexpressing Ovine $\beta$-Catenin. Int. J. Mol. Sci. 2019, 20, 620. [CrossRef]

7. Wu, P.; Zhang, Y.; Xing, Y.; Xu, W.; Guo, H.; Deng, F.; Ma, X.; Li, Y. The balance of Bmp6 and Wnt10b regulates the telogen-anagen transition of hair follicles. Cell Commun. Signal 2019, 17, 16. [CrossRef]

8. Song, L.L.; Cui, Y.; Yu, S.J.; Liu, P.G.; Liu, J.; Yang, X.; He, J.F.; Zhang, Q. Expression characteristics of BMP2, BMPR-IA and Noggin in different stages of hair follicle in yak skin. Gen. Comp. Endocrinol. 2018, 260, 18-24. [CrossRef] [PubMed]

9. Jin, M.; Zhang, J.Y.; Chu, M.X.; Piao, J.; Piao, J.A.; Zhao, F.Q. Cashmere growth control in Liaoning cashmere goat by ovarian carcinoma immunoreactive antigen-like protein 2 and decorin genes. Asian-Australas J. Anim. Sci. 2018, 31, 650-657. [CrossRef]

10. AlShaibi, H.F.; Ahmed, F.; Buckle, C.; Fowles, A.C.M.; Awlia, J.; Cecchini, M.G.; Eaton, C.L. The BMP antagonist Noggin is produced by osteoblasts in response to the presence of prostate cancer cells. Biotechnol. Appl. Biochem. 2018, 65, 407-418. [CrossRef]

11. Chen, G.; Xu, H.; Yao, Y.; Xu, T.; Yuan, M.; Zhang, X.; Lv, Z.; Wu, M. BMP Signaling in the Development and Regeneration of Cranium Bones and Maintenance of Calvarial Stem Cells. Front. Cell Dev. Biol. 2020, 8, 135. [CrossRef] [PubMed]

12. Jensen, G.S.; Leon-Palmer, N.E.; Townsend, K.L. Bone morphogenetic proteins (BMPs) in the central regulation of energy balance and adult neural plasticity. Metab. Clin. Exp. 2021, 123, 154837. [CrossRef]

13. Botchkarev, V.A.; Sharov, A.A. BMP signaling in the control of skin development and hair follicle growth. Differentiation 2004, 72, 512-526. [CrossRef]

14. Botchkarev, V.A. Bone morphogenetic proteins and their antagonists in skin and hair follicle biology. J. Invest. Dermatol. 2003, 120, 36-47. [CrossRef]

15. Wang, Z.; Chen, Y.; Chen, M.; Zhang, Y. Overexpression of Fgf8 in the epidermis inhibits hair follicle development. Exp. Dermatol. 2021, 30, 494-502. [CrossRef] [PubMed]

16. Sun, H.; Zhang, Y.; Bai, L.; Wang, Y.; Yang, L.; Su, W.; Gao, S. Heat stress decreased hair follicle population in rex rabbits. J. Anim. Physiol. Anim. Nutr. 2019, 103, 501-508. [CrossRef] [PubMed]

17. Noramly, S.; Morgan, B.A. BMPs mediate lateral inhibition at successive stages in feather tract development. Development 1998, 125, 3775-3787. [CrossRef]

18. Ceruti, J.M.; Oppenheimer, F.M.; Leirós, G.J.; Balañá, M.E. Androgens downregulate BMP2 impairing the inductive role of dermal papilla cells on hair follicle stem cells differentiation. Mol. Cell Endocrinol. 2021, 520, 111096. [CrossRef] [PubMed]

19. Cai, B.; Zheng, Y.; Yan, J.; Wang, J.; Liu, X.; Yin, G. BMP2-mediated PTEN enhancement promotes differentiation of hair follicle stem cells by inducing autophagy. Exp. Cell Res. 2019, 385, 111647. [CrossRef] [PubMed]

20. Esibizione, D.; Cui, C.Y.; Schlessinger, D. Candidate EDA targets revealed by expression profiling of primary keratinocytes from Tabby mutant mice. Gene 2008, 427, 42-46. [CrossRef] [PubMed]

21. Adly, M.A.; Assaf, H.A.; Hussein, M.R. Expression of bone morphogenetic protein-7 in human scalp skin and hair follicles. Br. J. Dermatol. 2006, 154, 551-554. [CrossRef] [PubMed]

22. Shimasaki, S.; Moore, R.K.; Otsuka, F.; Erickson, G.F. The bone morphogenetic protein system in mammalian reproduction. Endocr. Rev. 2004, 25, 72-101. [CrossRef] [PubMed]

23. Huang, H.J.; Wu, J.C.; Su, P.; Zhirnov, O.; Miller, W.L. A novel role for bone morphogenetic proteins in the synthesis of follicle-stimulating hormone. Endocrinology 2001, 142, 2275-2283. [CrossRef] [PubMed]

24. Glister, C.; Kemp, C.F.; Knight, P.G. Bone morphogenetic protein (BMP) ligands and receptors in bovine ovarian follicle cells: Actions of BMP-4, -6 and -7 on granulosa cells and differential modulation of Smad-1 phosphorylation by follistatin. Reproduction 2004, 127, 239-254. [CrossRef]

25. Erickson, G.F.; Fuqua, L.; Shimasaki, S. Analysis of spatial and temporal expression patterns of bone morphogenetic protein family members in the rat uterus over the estrous cycle. J. Endocrinol. 2004, 182, 203-217. [CrossRef]

26. Sun, W.; Ni, R.; Yin, J.F.; Musa, H.H.; Ding, T.; Chen, L. Genome array of hair follicle genes in lambskin with different patterns PLoS ONE 2013, 8, e68840. [CrossRef]

27. Lv, X.; Gao, W.; Jin, C.; Wang, L.; Wang, Y.; Chen, W.; Zou, S.; Huang, S.; Li, Z.; Wang, J.; et al. Preliminary study on microR-148a and microR-10a in dermal papilla cells of Hu sheep. BMC Genet. 2019, 20, 70. [CrossRef] [PubMed]

28. Lu, Z.; Du, L.; Liu, R.; Di, R.; Zhang, L.; Ma, Y.; Li, Q.; Liu, E.; Chu, M.; Wei, C. MiR-378 and BMP-Smad can influence the proliferation of sheep myoblast. Gene 2018, 674, 143-150. [CrossRef] [PubMed]

29. Livak, K.J.; Schmittgen, T.D. Analysis of relative gene expression data using real-time quantitative PCR and the 2(-Delta Delta C(T)) Method. Methods 2001, 25, 402-408. [CrossRef]

30. Hynd, P.I.; Edwards, N.M.; Hebart, M.; McDowall, M.; Clark, S. Wool fibre crimp is determined by mitotic asymmetry and position of final keratinisation and not ortho- and para-cortical cell segmentation. Animal 2009, 3, 838-843. [CrossRef]

31. Kwack, M.H.; Seo, C.H.; Gangadaran, P.; Ahn, B.C.; Kim, M.K.; Kim, J.C.; Sung, Y.K. Exosomes derived from human dermal papilla cells promote hair growth in cultured human hair follicles and augment the hair-inductive capacity of cultured dermal papilla spheres. Exp. Dermatol. 2019, 28, 854-857. [CrossRef]

32. Nissimov, J.N.; Das Chaudhuri, A.B. Hair curvature: A natural dialectic and review. Biol. Rev. Camb. Philos. Soc. 2014, 89, 723-766. [CrossRef]

33. Mishina, Y. Function of bone morphogenetic protein signaling during mouse development. Front. Biosci. 2003, 8, d855-d869. [CrossRef] [PubMed] 
34. Bai, W.L.; Dang, Y.L.; Wang, J.J.; Yin, R.H.; Wang, Z.Y.; Zhu, Y.B.; Cong, Y.Y.; Xue, H.L.; Deng, L.; Guo, D.; et al. Molecular characterization, expression and methylation status analysis of BMP4 gene in skin tissue of Liaoning cashmere goat during hair follicle cycle. Genetica 2016, 144, 457-467. [CrossRef] [PubMed]

35. Andl, T.; Ahn, K.; Kairo, A.; Chu, E.Y.; Wine-Lee, L.; Reddy, S.T.; Croft, N.J.; Cebra-Thomas, J.A.; Metzger, D.; Chambon, P.; et al. Epithelial Bmpr1a regulates differentiation and proliferation in postnatal hair follicles and is essential for tooth development. Development 2004, 131, 2257-2268. [CrossRef]

36. Jamora, C.; DasGupta, R.; Kocieniewski, P.; Fuchs, E. Links between signal transduction, transcription and adhesion in epithelial bud development. Nature 2003, 422, 317-322. [CrossRef]

37. Takahashi, H.; Ikeda, T. Transcripts for two members of the transforming growth factor-beta superfamily BMP-3 and BMP-7 are expressed in developing rat embryos. Dev. Dyn. 1996, 207, 439-449. [CrossRef]

38. Zouvelou, V.; Luder, H.U.; Mitsiadis, T.A.; Graf, D. Deletion of BMP7 affects the development of bones, teeth, and other ectodermal appendages of the orofacial complex. J. Exp. Zool. B Mol. Dev. Evol. 2009, 312B, 361-374. [CrossRef]

39. Song, D.; Liu, X.; Liu, R.; Yang, L.; Zuo, J.; Liu, W. Connexin 43 hemichannel regulates H9c2 cell proliferation by modulating intracellular ATP and [Ca ${ }^{2+}$. Acta Biochim. Biophys. Sin. 2010, 42, 472-482. [CrossRef] [PubMed]

40. Han, Y.; Zhang, P.J.; Chen, T.; Yum, S.W.; Pasha, T.; Furth, E.E. Connexin43 Expression Increases in the Epithelium and Stroma along the Colonic Neoplastic Progression Pathway: Implications for Its Oncogenic Role. Gastroenterol. Res. Pract. 2011, 2011, 561719. [CrossRef]

41. Medland, S.E.; Nyholt, D.R.; Painter, J.N.; McEvoy, B.P.; McRae, A.F.; Zhu, G.; Gordon, S.D.; Ferreira, M.A.; Wright, M.J.; Henders, A.K.; et al. Common variants in the trichohyalin gene are associated with straight hair in Europeans. Am. J. Hum. Genet. 2009, 85, 750-755. [CrossRef] [PubMed]

42. Ellis, T.; Gambardella, L.; Horcher, M.; Tschanz, S.; Capol, J.; Bertram, P.; Jochum, W.; Barrandon, Y.; Busslinger, M. The transcriptional repressor CDP (Cutl1) is essential for epithelial cell differentiation of the lung and the hair follicle. Genes Dev. 2001, 15, 2307-2319. [CrossRef] [PubMed]

43. Zeisberg, M.; Hanai, J.; Sugimoto, H.; Mammoto, T.; Charytan, D.; Strutz, F.; Kalluri, R. BMP-7 counteracts TGF-beta1-induced epithelial-to-mesenchymal transition and reverses chronic renal injury. Nat. Med. 2003, 9, 964-968. [CrossRef] [PubMed] 